\title{
Ações corporificadas e construção de turnos em uma interação entre terapeuta e criança com Transtorno do Espectro Autista (TEA)
}

DOI: http://dx.doi.org/10.21165/el.v50i1.2912

\author{
Fernanda Miranda da Cruz ${ }^{1}$ \\ Ana Caroline Lopes Gomes Guerra² \\ Ana Carina Tamanaha ${ }^{3}$ \\ Jacy Perissinoto 4
}

\section{Resumo}

Os objetivos deste trabalho são explorar como algumas ações interacionais corporificadas silenciosas são construídas, contribuindo para a descrição de interações envolvendo sujeitos com Transtornos do Espectro do Autismo (TEA). Transcrevemos e descrevemos quatro sequências em que perguntas e solicitações verbais de uma terapeuta são dirigidas a uma criança com TEA e respondidas por ações corporificadas não-verbais. Identificamos algumas formas pelas quais os interlocutores constroem, monitoram e gerenciam as múltiplas e complexas temporalidades de uma interação que envolvem ações de: a) projeções; b) sequencialidade e c) inteligibilidade de unidades constitutivas dos turnos. As análises apresentadas colaboraram para o refinamento de métodos qualitativos para a descrição da dinâmica interacional de interações envolvendo sujeitos com TEA.

Palavras-chaves: Transtornos do Espectro do Autismo; temporalidade; ações corporificadas.

1 Universidade Federal de São Paulo (UNIFESP), São Paulo, São Paulo, Brasil; fernanda.miranda.cruz@gmail.com; https://orcid.org/0000-0002-5051-7759

2 Universidade Federal de São Paulo (UNIFESP), São Paulo, São Paulo, Brasil; anaguerra.ac@gmail.com; https://orcid.org/0000-0003-1104-1732

3 Universidade Federal de São Paulo (UNIFESP), São Paulo, São Paulo, Brasil; anacarinatamanaha@gmail.com; https://orcid.org/0000-0001-9915-6299

4 Universidade Federal de São Paulo (UNIFESP), São Paulo, São Paulo, Brasil; jacydch.otor@epm.br; https://orcid.org/0000-0002-0287-9296 


\title{
Embodied actions and turn-taking in an interaction between therapist and child with Autistic Spectrum Disorder (ASD)
}

\begin{abstract}
This article aims to explore how some silent embodied interactional actions are constructed to contribute to the descriptions of interactions with people with Autism Spectrum Disorders (ASD). We transcribed and described four sequences in which verbal questions are addressed to a child with ASD and answered by embodied non-verbal actions. We identified some ways in which the participants accomplish, monitor, and manage the multiple and complex temporalities of an interaction that involve actions of a) projections, b) sequentially, and c) intelligibility of turns' construction units. This analysis contributes to the improvement of qualitative methods for the description of the interactional dynamics of atypical interactions.
\end{abstract}

Keywords: Autism Spectrum Disorder; temporality; embodiment action.

\section{Introdução}

O presente estudo tem como objetivo central explorar como algumas ações interacionais corporificadas silenciosas são construídas e contribuir com as descrições de interações envolvendo sujeitos com Transtornos do Espectro do Autismo (TEA), colaborando para o refinamento de métodos qualitativos para a descrição da dinâmica interacional de interações atípicas. O TEA é compreendido, clinicamente, como uma condição congênita que afeta o desenvolvimento neurocognitivo. Apesar de ser um grupo bastante heterogêneo de características e sintomas, as características principais do TEA são prejuízos na comunicação e na interação social; dificuldade na construção de atenção conjunta e na participação em interações sociais (LAl; LOMBARDO; BARON-COHEN, 2013; CUNHA; BORDINI; CAETANO, 2015; TAMANAHA; PERISSINOTO, 2019).

Analisaremos interações com uma estrutura sequencial específica: sequências em que perguntas ou solicitações verbais de um adulto dirigidas a uma criança com TEA são seguidas de silêncio verbal. Essetipo de sequência não éexclusivo de interações envolvendo sujeitos com TEA. Tampouco as respostas em silêncio significam necessariamente não responsividade interacional de crianças com TEA. Sequências com essa organização, em que um turno verbal dirigido ao outro seguido por silêncio verbal ou respostas silenciosas, foram identificadas, por exemplo, em sequências interacionais de instrução, solicitação ou diretivas (MONDADA, 2014b, GOODWIN; CEKAITE, 2014). Essas interações são sequencialmente organizadas como pares de adjacência, em que a primeira parte do par pode ser reconhecida como uma instrução ou diretiva (GOODWIN, 2006; MONDADA, 2011). Essa primeira parte estabelece uma relevância condicional para a segunda parte do par, que seria a resposta interacional (GOODWIN, 2006) ou a própria realização da ação (MONDADA, 2011). Essas respostas silenciosas podem ser estruturadas por ações corporificadas relevantes (LINDWALL; EKSTRÖM, 2012; MONDADA, 2009, 2011). 
A pertinência analítica desse tipo de sequência está naanálise do que ocorre corporalmente nesta segunda parte silenciosa de um par adjacente, que pode revelar um conjunto de ações corporificadas interacionais em silêncio, sugerindo que uma qualificação de critérios para o grau de participação de uma criança com TEA em interações, seja em ambiente familiar, escolar ou terapêutico, pode se beneficiar de uma maior sistematização descritiva de quais pistas de participação interacional se mostram relevantes.

Nossas análises foram informadas pelos métodos e procedimentos do campo dos estudos interacionais multimodais (GOODWIN, 1986; ERICKSON; SCHULTZ, 1982; HEATH, 1986; MONDADA, 2016a), incluindo os estudos aplicados a interações envolvendo autistas (KORKIAKANGAS, 2014; DOAK, 2018). Essa abordagem considera não somente o papel da linguagem verbal na construção das interações, mas também dos aspectos nãoverbais e mostra como prosódia, posturas corporais, gesto, expressão facial, por exemplo, nos fornecem uma grande quantidade de informações a respeito da organização e da estruturação da própria interação. Esses recursos podem ser mais convencionalizados, tais como os recursos linguísticos e alguns recursos gestuais, ou mais improvisados e contingentes (MONDADA, 2019), incluindo vocalizações, movimentos e manipulação de objetos, que são altamente dependentes das especificidades do contexto (MONDADA, 2019). Os aspectos não-verbais estariam não apenas coordenados com a fala (KENDON, 2004), mas também configuram, juntamente com os aspectos verbais, o fluxo conversacional, a sequencialidade e a temporalidade das interações (MONDADA, 2004).

Analisaremos um pequeno conjunto de quatro momentos de uma interação entre uma terapeuta e uma criança com TEA. Esta última é verbal, considerada, clinicamente, um caso de TEA de bom funcionamento. A análise parte da premissa de que, nas interações, os participantes são instados a conduzir suas ações dentro de regras de temporalidade e de sequencialidade de uma dinâmica interacional que envolve uma Gestalt Multimodal Complexa (MONDADA, 2016a), caracterizada por distintas projeções, temporalidades e trajetórias de microações constitutivas de nossas interações com o outro. Ao fazermos uma análise multimodal de interações, conseguimos identificar detalhes das formas pelas quais os interlocutores constroem, monitoram e gerenciam as múltiplas e complexas temporalidades de uma interação que envolvem ações de a) projeção; b) sequencialidade; c) inteligibilidade dos potenciais locais de transição de turno (LRT) e das unidades de construção de turno (UCT); e d) construção da interação por ações corporificadas nãoverbais.

\section{Temporalidade e sequencialidade das interações}

A dinâmica de trocas de turnos de fala tem sido amplamente estudada (SACKS; SCHEGLOFF; JEFFERSON, 1974; MARCUSCHI, 1991; SILVA; ANDRADE; OSTERMANN, 2009; LODER; SALIMEN; MULLER, 2008). Do ponto de vista estrutural, os turnos de fala são constituídos por "segmentos construídos a partir de unidades de construção de turno (UCT) e podem corresponder a unidades como sentenças, orações, palavras 
isoladas, locuções frasais ou recursos prosódicos." (JUNG; MAREGA, 2011, p. 323). Essas unidades são "determinadas por traços da estrutura linguística superficial" (LEVINSON, 2007, p. 377), portanto, da fala em uso. Dessa forma, as UCT são unidades que possuem uma composição lexical, sintática, prosódica e sua completude se dá de forma situada e contingente pelas dinâmicas interacionais entre os participantes (LEVINSON, 2007).

No curso de uma interação, geralmente, há uma dinâmica de alternância de turnos entre os interlocutores que ocorre ao fim de uma UCT, incluindo as possíveis sobreposições de turnos que parecem não ocorrer em qualquer momento na interação (LEVINSON, 2007; OLLOF, 2013). O final de cada UCT é um ponto potencial para a alternância do turno, sendo um local de relevância de transição (LRT). Essa estrutura organizacional indica que o processo de alternância de turno requer uma identificação de aspectos de projeção ou previsibilidade do fim de cada UCT. A descrição das dinâmicas de trocas de turnos verbais tem ganhado outras direções com os estudos multimodais (AUER, 2005; STUKENBROCK, 2014; MONDADA, 2019; dentre outros). Por exemplo, tem sido interessante mostrar, a partir de uma perspectiva multimodal, que locais relevantes de transição de turno podem ser mobilizados ou ocupados tanto por recursos verbais quanto por recursos não-verbais. Essa projeção diz respeito à possibilidade de os participantes poderem prever, durante uma UCT em curso, qual tipo de unidade está sendo produzida e em que momento ela potencialmente se completa. A própria definição de turno tem sido revista a partir da consideração dos aspectos não-verbais constitutivos da dinâmica interacional (DEPPERMANN, 2013). Neste artigo, uma revisão bibliográfica mais robusta da crescente e atual literatura sobre a noção de turno a partir da perspectiva multimodal (MONDADA, 2008; DEPPERMANN, 2013) não foi feita.

As ações de identificação, monitoramento e gerenciamento das UCT e dos LRT realizadas pelos participantes durante a construção de suas interações são pertinentes para dar visibilidade às microações de coordenação e colaboração envolvendo a participação, mesmo que silenciosa, de sujeitos com TEA em interações com o outro. Como aponta Mondada (2019, p. 2, tradução nossa5), a respeito do tratamento analítico do silêncio nos estudos conversacionais:

5 No original: "Within Conversation Analyis, silence has been categorized in different ways, as the absence of talk at different sequential locations with respect to the organization of turns (Hoey, 2017). An intra-turn silence occurring in the middle of a turn, before its completion and before a transition-relevance point TRP is reached, is a pause; an inter-turn silence that occurs within a TRP is a gap; and an extended silence after a TRP is a lapse. This terminology is not straightforward when considering actions that are silently achieved: for example, when considering embodied compliance to a directive, it is problematic to treat the second pair part as occurring within a gap or a lapse. This shows that silence is not always the absence of talk. Silence invites us to consider the temporality of embodied actions more closely". 
Na Análise da Conversa, o silêncio foi categorizado de diferentes maneiras, como a ausência de fala em diferentes locais sequenciais no que diz respeito à organização dos turnos (Hoey, 2017). Um silêncio intra-turno ocorrendo no meio de um turno, antes de sua conclusão e antes de um local relevante de transição LRT ser alcançado, é uma pausa; um silêncio entre turnos que ocorre dentro de um LRT é uma lacuna; e um silêncio prolongado após um LRT é um lapso. Essa terminologia não se aplica ao considerarmos as ações que são realizadas silenciosamente: por exemplo, ao considerar a resposta corporificada a uma diretiva, é problemático tratar a segunda parte do par como ocorrendo dentro de uma lacuna ou lapso. Isso mostra que o silêncio nem sempre é a ausência de fala. O silêncio nos convida a considerar mais de perto a temporalidade das ações corporificadas.

Para Mondada (2019), dentre um conjunto de questões da agenda dos estudos linguísticos, está a pergunta em torno de como as ações corporificadas e os recursos verbais se distribuem em uma atividade situada, quando momentos de fala são alternados com momentos de ações em silêncio.

\section{Métodos e materiais}

Visualizamos 10 horas de vídeo de interações do corpus audiovisual de interações entre terapeutas-crianças-familiares (Comitê de Ética em Pesquisas - CAAE 59128416.3.0000.5505). Os registros foram gerados pelo Núcleo de Investigação Fonoaudiológica em Linguagem de Crianças e Adolescentes com Transtornos do Espectro Autista (NIFLINC-TEA) do Departamento de Fonoaudiologia da Universidade Federal de São Paulo (UNIFESP), sob a responsabilidade das pesquisadoras Ana Carina Tamanaha e Jacy Perissinoto. Selecionamos os dados de uma interação em particular entre Enrique (ENR), com diagnóstico de TEA, e a fonoaudióloga Clara (CLA). Essa interação teve uma duração de 3 minutos e 13 segundos e corresponde a momentos de uma atividade de imitação proposta pela terapeuta.

Enrique nasceu em fevereiro de 2008. Um relato da mãe feito ao serviço de atendimento, quando Enrique tinha 4 anos e 7 meses, descreve "ele não consegue me explicar nada, não entende as orientações dadas na escola, temos que falar as coisas várias vezes e nem sempre ele entende". Em outro relato posterior, com 6 anos, a mãe considerava que Enrique estava bem, "mas, às vezes, fala coisas fora de hora". Em uma avaliação feita com a idade de 6 anos e 7 meses, alguns aspectos interacionais foram destacados: restrição na manutenção do contato visual; mas alinhamento às regras de troca de turnos, ainda que com poucas iniciativas de fala; dificuldade na compreensão de ordens simples na conversa espontânea, que era facilitada quando contava com apoio de figuras e gestos; dificuldade na compreensão de inferências; dificuldade na elaboração de relatos e recontos e ocorrências de ecolalia imediata e tardia. Na interação analisada, 
Enrique tinha 7 anos e 1 mês. Nos interessa destacar, na descrição do perfil interacional de Enrique, a identificação de sua compreensão e uso das regras de troca de turnos. Vale lembrar que Enrique é um autista verbal, com necessidade de pouco suporte do outro para suas atividades. Respeitar as regras de turno é um indicador de uma inteligibilidade das unidades de constituição de turno, uma vez que exige a identificação e o monitoramento das mesmas. Nosso enfoque de análise recairá especificamente sobre os traços entonacionais, os direcionamentos de olhar e as mudanças de posturas corporais que indicam a inteligibilidade e, portanto, identificação e monitoramento das unidades de constituição de turno. Antecipamos uma limitação deste artigo. A análise empreendida neste artigo se restringiu a uma interação envolvendo uma criança autista verbal. No entanto, uma análise como esta futuramente direcionada a interações com autistas não-verbais nos parece produtiva e necessária.

A terapeuta, antes do início do teste de imitação, faz uma instrução verbal sobre o que deve ser feito: imitar o que ela vai fazer. Os dois participantes estão sentados um diante do outro em uma mesa. A câmera tem um ângulo que apreende a interação global, conforme Figura 1 abaixo.

Figura 1. Terapeuta mostra uma ação a ser imitada pela criança

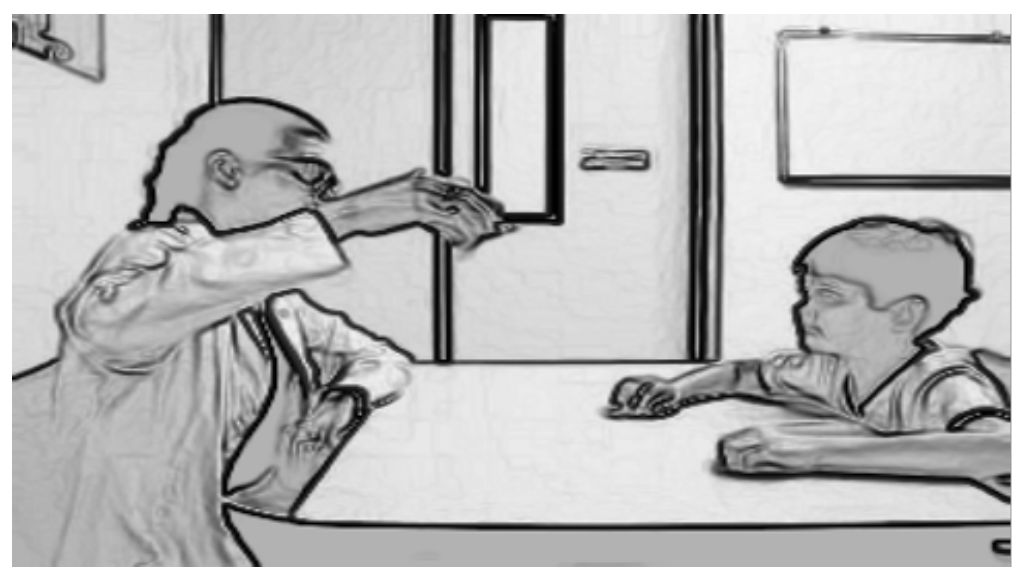

Fonte: Elaboração própria

Para transcrição dos dados, adotamos o sistema de convenção de Mondada (2016b), disponível em: https://bit.ly/3xqnDqD. Essa convenção ébaseada no princípio fundamental de que todas as ações corporificadas estão precisamente localizadas no tempo e são coordenadas entre si. A coordenação dessas ações não significa necessariamente simultaneidade, mas sincronia e ajuste com as ações do outro e com a temporalidade de toda a interação. Como tem demonstrado Mondada (2019), a localização precisa das ações corporificadas por meio da transcrição permite proceder a uma análise de quão responsivos os participantes são uns aos outros e, portanto, permite a identificação de qual participante, com qual recurso, iniciou qual ação. A visualização e a transcrição dos dados foi realizada com auxílio do software ELAN (WITTENBURG et al., 2006). 


\section{Temporalidades complexas em uma atividade de imitação entre uma terapeuta e uma criança com TEA}

Ao longo da interação, a terapeuta pega objetos para utilizar durante o teste (moto de brinquedo, escova de dentes, vela, sapo de brinquedo). Do ponto de vista de uma organização pragmática, o teste de imitação acontece dentro de uma dinâmica de alternância entre os interlocutores a partir de três ações (ora corporificadas, ora verbais) que se repetem a cada nova rodada: ação 1 (a terapeuta demonstra uma ação corporificada em silêncio a ser imitada pela criança, por exemplo, fazer um sapo pular, escovar o dente); ação 2 (a terapeuta passa o turno à criança, através de um ação verbal, "sua vez"); ação 3 (a criança imita através de uma ação corporificada, em silêncio, a demonstração inicial feita pela terapeuta). Do ponto de vista interacional, muitas outras microações silenciosas acontecem entre as três etapas de ações identificadas acima e dizem respeito à forma como Enrique exibe seu entendimento do que deve ser feito; à forma como os dois participantes fazem a transição do turno de um para o outro, como marcam a trajetória de suas ações e como sinalizam sua finalização.

Analisamos quatro momentos dessa interação. No excerto 1, a terapeuta mostra uma moto de brinquedo para a criança (linha 04) e começa a demonstrar o movimento da moto andando (linha 07) enquanto, simultaneamente, vocaliza o barulho do motor (linha 09). Enquanto realiza essas ações de demonstração e vocalização da moto, Enrique acompanha com olhar a demonstração do movimento da moto com a cabeça (linha 02). A terapeuta finaliza a ação de imitar demonstrativa do que Enrique deve fazer em seguida, na linha 15, e olha para Enrique. Logo após o olhar mútuo entre os dois, a terapeuta Ihe entrega o brinquedo para iniciar a imitação.

Queremos evidenciar o momento em que Enrique inicia uma mudança de postura na cadeira, iniciando a preparação do seu turno de ação corporificado (imitar) e indicando a projeção do fim do turno da avaliadora, o que evidencia seu monitoramento da construção do turno de sua interlocutora. Essa preparação se inicia na linha 12, antes da passagem verbal do turno (linha 18) feita pela terapeuta, o que indica uma projeção da completude do turno da terapeuta orientada pela entonação descendente da vocalização "vrum".

Excerto 1 - Imitar uma moto andando

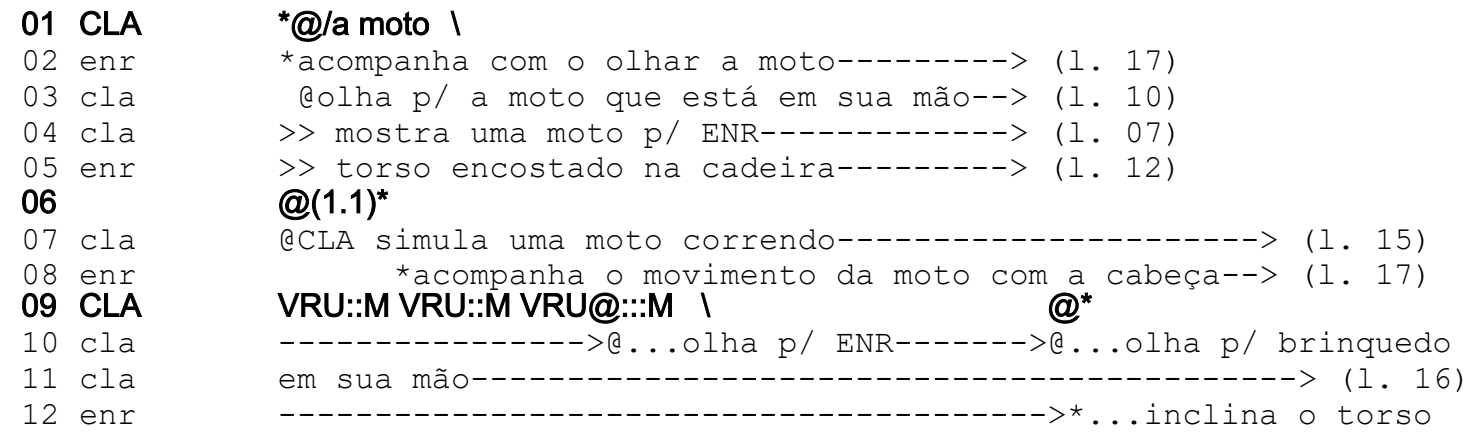




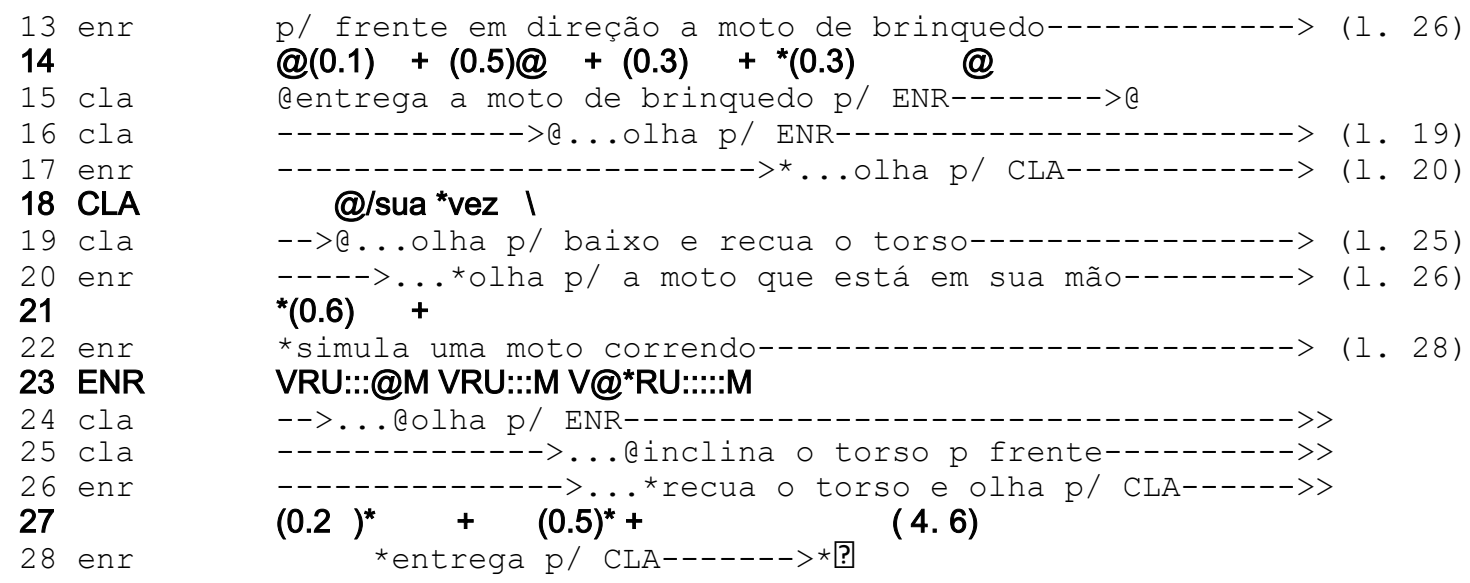

A entonação descendente é um traço de finalização de uma UCT e uma potencial LRT. Enrique projeta o curso e a finalização dessa UCT uma vez que se orienta para esses traços de finalização do turno e inclina o torso para frente em direção a moto (linha 12). Ainda que saibamos, pela configuração situada de um teste sendo aplicado por uma terapeuta adulta a uma criança paciente com TEA, que a terapeuta conduz a interação, também podemos notar que os ajustes e as dinâmicas da temporalidade dessa interação são coordenados pelos dois participantes. Enrique, ao mudar sua postura corporal e aproximar-se do campo visual da terapeuta, indica ou torna visível seu entendimento do que deve ser feito. Da mesma forma, essa ação corporificada orienta a próxima ação da terapeuta. Há um período de silêncio verbal (1.2 segundos) e ambos, terapeuta e criança, se olham mutuamente. Em um estudo realizado por Bavelas, Coates e Johnson (2002) em ambiente experimental, algumas funções do olhar mútuo foram identificadas, como na transição de falantes e na solicitação de respostas: "O ouvinte tendia a responder quando o locutor olhava para ela, e o locutor tendia a desviar o olhar logo após o ouvinte responder. Juntos, falantes e ouvintes criaram e usaram a janela do olhar [olhar mútuo] para coordenar suas ações" (ROSSANO, 2013, p. 309, tradução nossa).

No excerto acima, essa janela do olhar ocorre ao final da ação 1 (demonstração do que deve ser imitado), o que publiciza alguns entendimentos específicos de uma sequência de ações: a finalização da ação de demonstração e o entendimento do curso da ação. Da mesma forma, no excerto 2, a orientação de Enrique para a projeção da completude do turno se volta para a entonação descendente das vocalizações "PUM PUM PUM" (linha 14), referentes à ação de imitar um sapo pulando.

Excerto 2 - Imitar um sapo pulando na mesa

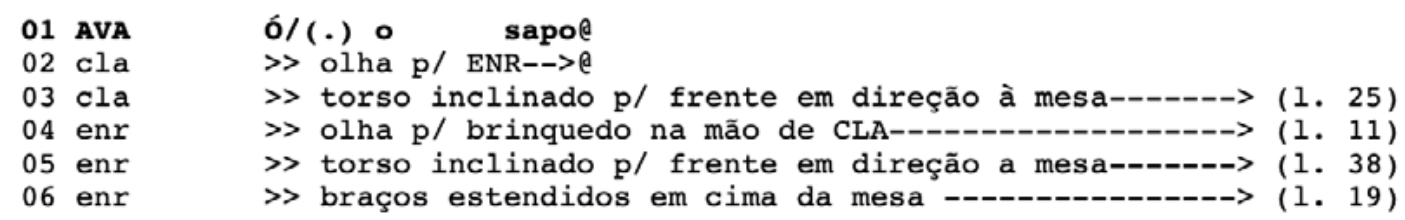




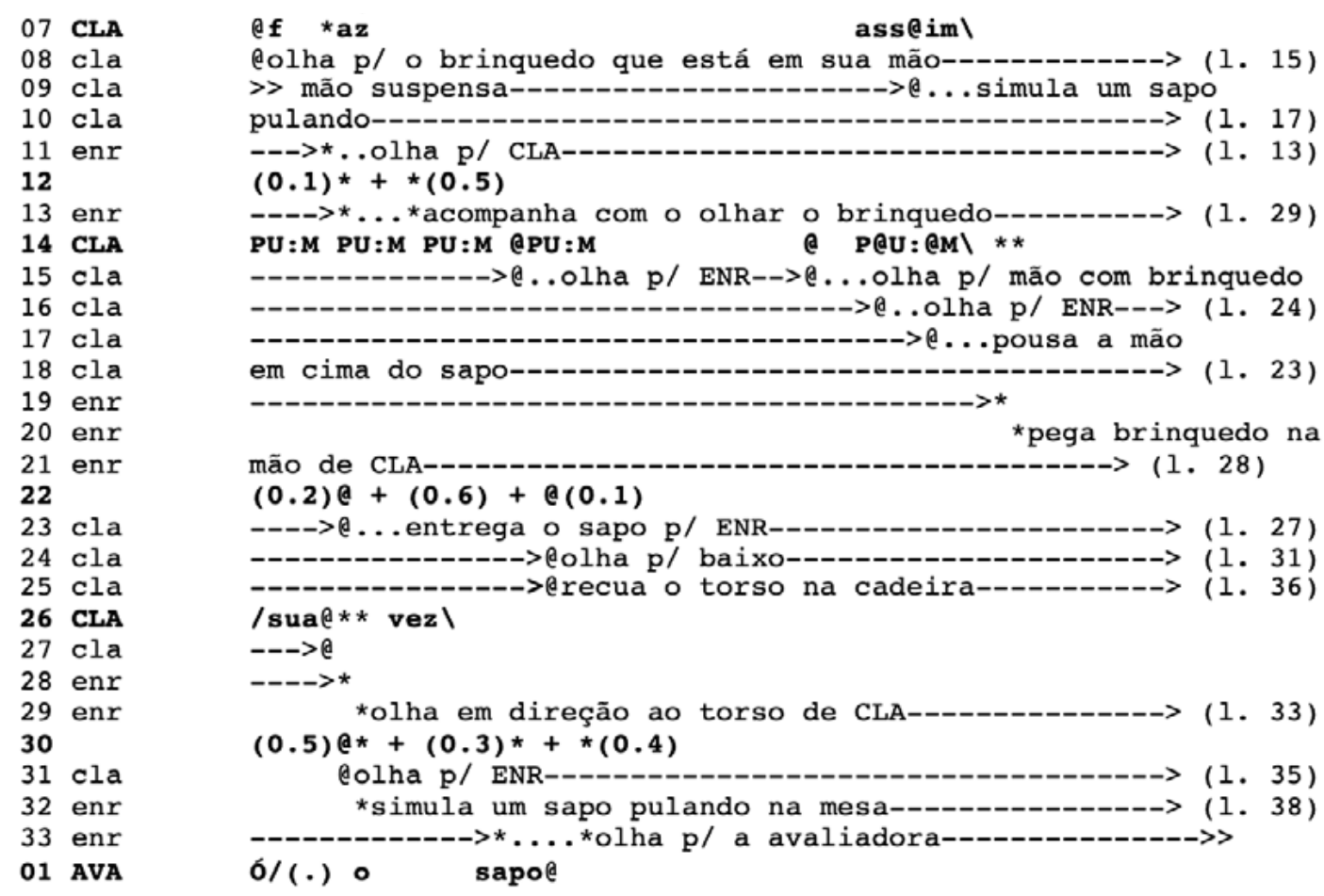

Nesse segundo excerto, a terapeuta, ao terminar de vocalizar o último "PUM" (linha 14), com entonação descendente, olha para a criança no momento em que pousa a mão em cima do sapo (linha 16 e 17), sinalizando que é o fim de uma UCT e abrindo espaço para um LRT, ou seja, uma possibilidade de alternância de turno. A criança, logo ao final dessa UCT, como já estava com o torso inclinado para frente, estende a mão para frente para pegar o brinquedo na mão da avaliadora, indicado na linha 20. E na linha 23, a terapeuta entrega o brinquedo para a criança. Em seguida, há um silêncio verbal e só então há a marcação verbal de troca de turno (linha 26).

A preparação da tomada de turno, tanto no excerto 1 como no excerto 2, indica uma inteligibilidade por parte dos participantes das unidades mínimas que o compõem. 0 sistema de trocas de turnos exige unidades mínimas sobre as quais operar (LEVINSON, 2007). Essas unidades são determinadas por vários traços da estrutura linguística superficial: são unidades sintáticas (sentenças, orações, sintagmas nominais etc.), identificadas como unidades de turno em parte por meios prosódicos e, especialmente, por meios entonacionais (LEVINSON, 2007). O conceito de projeção tem sido estudado principalmente com foco em unidades de ação ou em elementos linguístico-gramaticais (AUER, 2005). Como aponta Stukenbrock (2014), a projeção opera em diferentes domínios, que vão desde microfenômenos no nível fonológico (segmental) e prosódico, até componentes no nível da morfologia, léxico-semântica e sintaxe. Mas os fenômenos corporificados não parecem periféricos nas ações de projeção. 
No caso das vocalizações "vrum vrum vrum" e "PUM PUM PUM", não temos exatamente uma sentença sintática. Keevallik (2005) enfoca o fenômeno das demonstrações vocais e corporais. A autora investiga a construção de multiunidades de turnos em que certas configurações corpóreo-visuais produzidas em conjunção com vocalizações não lexicais, (a) podem acompanhar a fala na construção de uma UCT; (b) pode completar uma UCT iniciada por sintaxe na fala; ou (c) em última instância constituem uma UCT por conta própria. Keevallik (2005) considera e analisa essas configurações como padrões emergentes que não priorizam a linguagem e a fala, categorizando-as como unidades sintático-corporais. No caso das produções "vrum" e "PUM", o que nos interessa é justamente o fato de termos nessas sequências vocalizadas o emprego do traço de entonação descendente que indica um curso de finalização da ação de demonstração. Esse traço é interpretado por Enrique como projeção de finalização da construção do turno, tornando condicionalmente relevante sua preparação para o início de sua resposta corporificada.

A ideia de projeção também opera sobre outras dimensões corporificadas, em que o comportamento corporal visível desempenha um papel crucial em prever coisas ou ações que estão por vir e preparar as próximas ações (STUKENBROCK, 2014). Assim, além da entonação, marcada nas vocalizações (vrum e PUM), também destacamos outras ações corporificadas que permitem dar visibilidade às temporalidades coordenadas: inclinação do corpo, direcionamentos do olhar e o ritmo de repetições das ações demonstrativas de imitação, como assoprar a vela (excerto 3) e escovar dente (excerto 4).

Excerto 3 - Assoprar uma vela

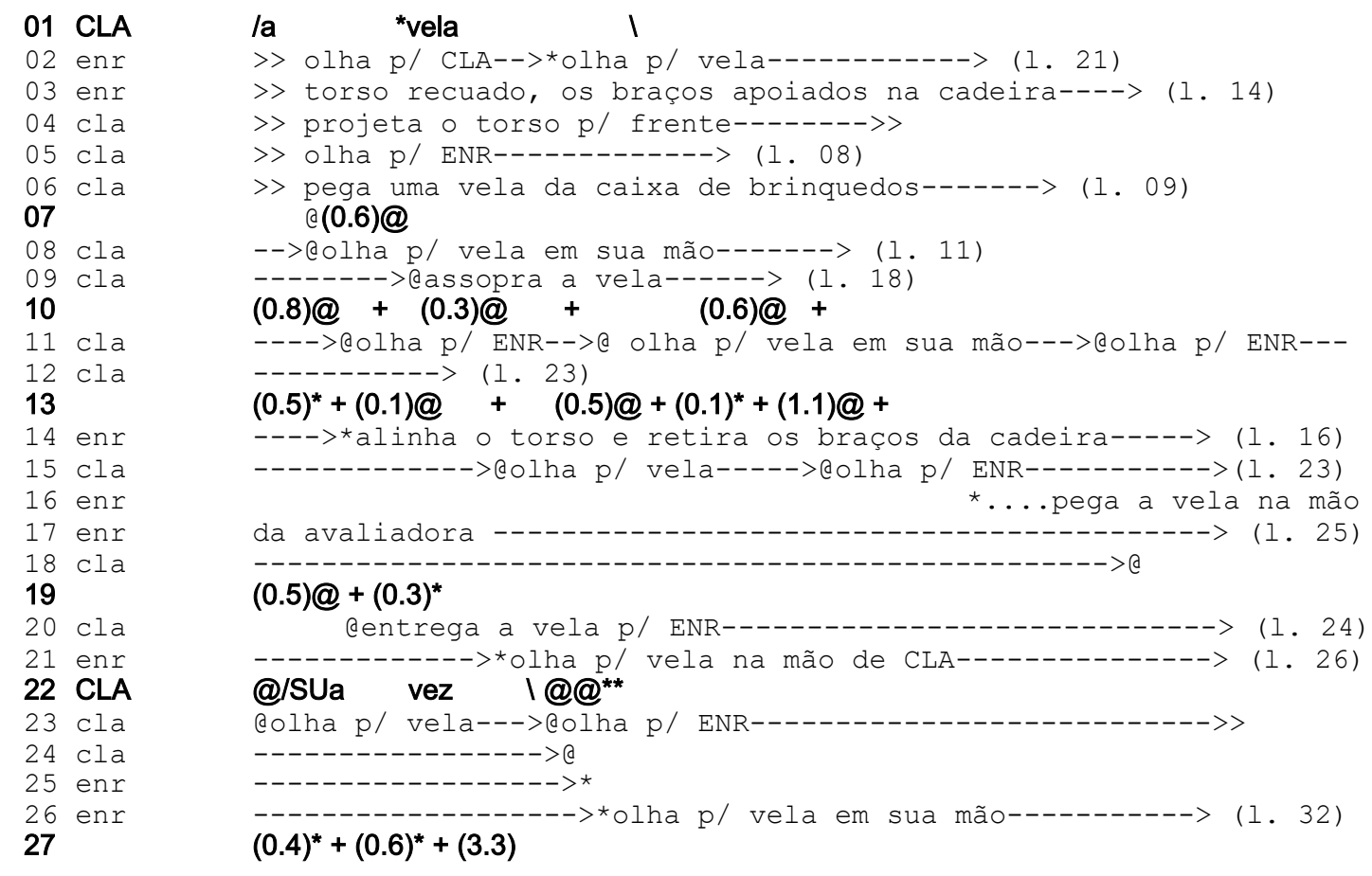




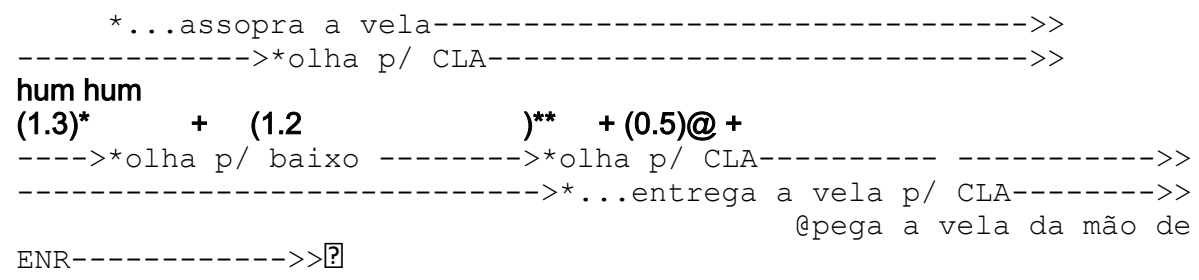

Nesse excerto, a terapeuta começa a demonstrar a ação de soprar a vela (linha 09) e finaliza essa ação só na linha 18. Ela realiza verbalmente a troca de turno apenas na linha 22, "sua vez". Mas, ao nos atentarmos para os movimentos de Enrique, percebemos que muito antes, na linha 14, Enrique se orienta para o fim do turno da terapeuta, ajustando seu torso na cadeira e retirando os braços da cadeira, no momento em que a avaliadora direciona o olhar para ele ao final das ações de assoprar a vela (linhas 11 a 15). O olhar pode ter muitas funções na interação, por exemplo, regulador da dinâmica de turno (ROSSANO, 2013).

Em um quarto excerto, a terapeuta pega um novo objeto, uma escova de dentes. Ela começa a demonstrar uma escovação enquanto olha para Enrique. Ao terminar a demonstração da imitação, entrega a escova, diz "sua vez" e Enrique repete os movimentos realizados anteriormente pela terapeuta. Mas Enrique inicia sua preparação para realizar a ação de imitação e indica a projetação da completude da solicitação da avaliadora ao final no curso da ação de demonstração "escovar dente". Ao inclinar seu corpo, Enrique sinaliza seu entendimento do que é para fazer e sua disponibilidade para iniciar seu turno de imitação. Essa sequência deixa de forma bem visível que, embora seja a terapeuta quem conduz a atividade, as temporalidades de quem inicia as ações são mais complexas. Tanto a terapeuta quanto Enrique ajustam a temporalidade de suas ações a esses pequenos movimentos corporais de cada um deles.

Excerto 4 - Imitar a ação de escovar o dente
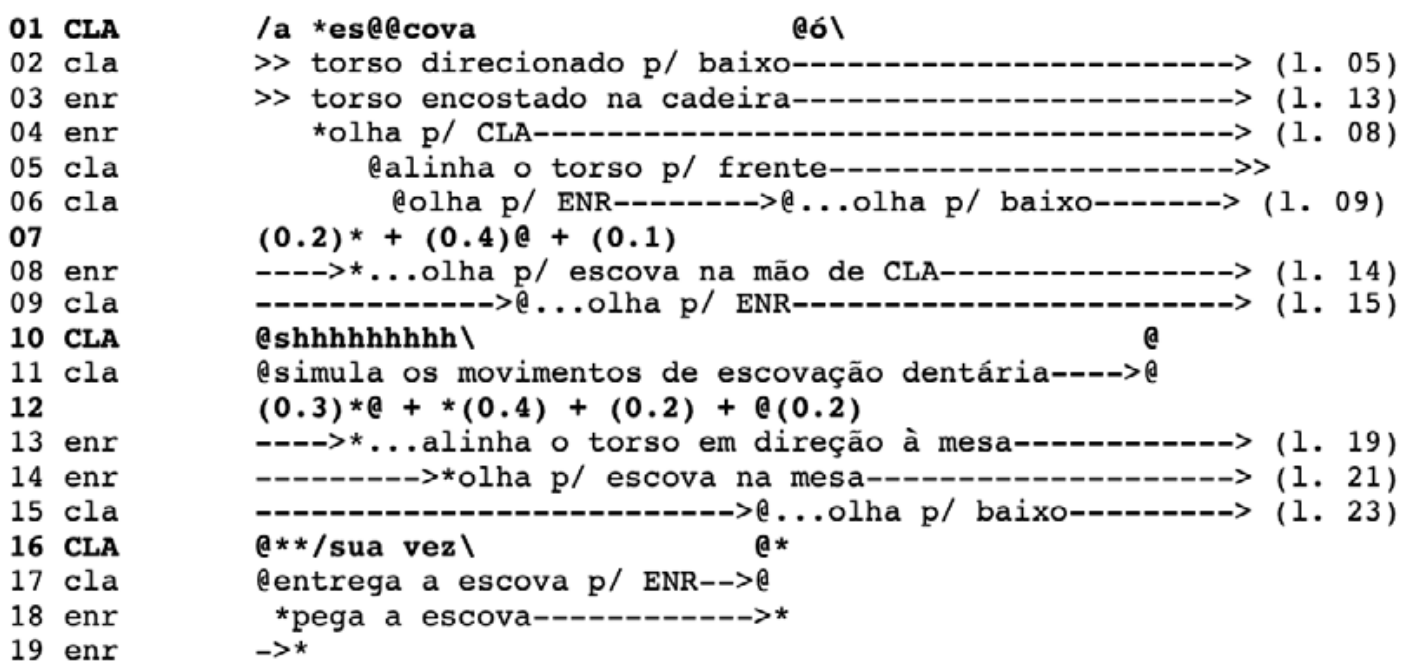


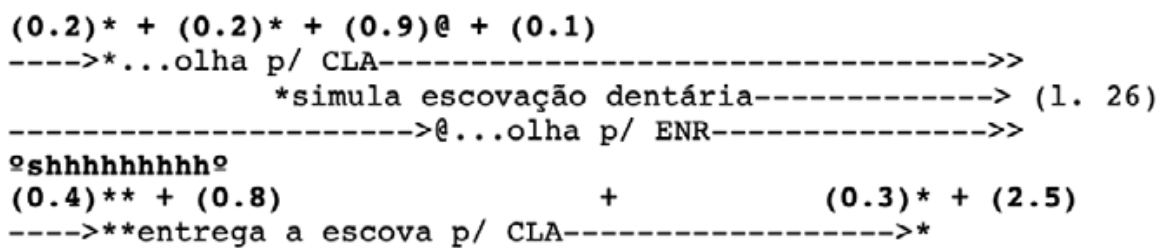

Nos quatro excertos, a proposta de imitação da terapeuta é coordenada com as ações de Enrique, como a preparação para iniciar o turno de imitação por mudança de postura corporal (recurso não-verbal) orientando-se para as pistas da estrutura superficial da fala, por exemplo, a entonação descendente das vocalizações (vrum e PUM dos excertos 1 e 2), e o direcionamento do olhar da avaliadora ao final das demonstrações (excertos 3 e 4). Essas microações publicizam alguns entendimentos específicos dessa sequência de ações: a finalização da ação de demonstração, entendimento do curso da ação, o momento em que efetivamente Enrique deve iniciar sua resposta ao teste (sua vez de iniciar a sequência de imitações).

\section{Discussão}

Ao nos atentarmos para as microações que constituem a organização sequencial do teste de imitação, além das três ações (ora corporificadas, ora verbais) que se repetem a cada nova rodada, ou seja, a ação 1 (a terapeuta demonstra uma ação corporificada em silêncio a ser imitada por Enrique); ação 2 (a terapeuta passa o turno a Enrique); ação 3 (Enrique imita através de uma ação corporificada, em silêncio, a demonstração inicial feita pela terapeuta), identificamos, através de aspectos não-verbais, que Enrique modula uma resposta inicial à solicitação da terapeuta antes mesmo desta última passar verbalmente o turno (ação 2), indicando que identifica e projeta a finalização da ação de imitar demonstrada pela terapeuta (ação 1) e se coloca disponível para iniciar seu papel na interação de realizar ou cumprir (LINDWALL; EKSTRÖM, 2012; MONDADA, 2009, 2011) a imitação. Essas microações deixam ver o que acontece no substrato interacional (MARLAIRE; MAYNARD, 1990) em que o teste de imitação ocorre. Para além das habilidades de imitar ou não as proposições da terapeuta, temos em jogo as habilidades de coordenar as múltiplas temporalidades de uma interação social em que, neste conjunto de dados do artigo, acontecem com a participação verbal da terapeuta e a participação corporificada silenciosa da criança com TEA. Vale lembrar que Enrique faz uso da linguagem verbal em suas outras interações. Optamos por trazer um caso em que as habilidades interacionais e respeito às regras de trocas de turnos já haviam sido identificadas e avaliadas por profissionais fonoaudiólogos, justamente por considerar que a metodologia e análise empreendidas aqui podem ser estendidas a casos de interações de sujeitos com TEA com comprometimentos dessa dimensão e, neste caso, relevar como se dão as dificuldades de monitoramento e identificação das unidades constitutivas da dinâmica interacional, incluindo as ausências ou formas compensatórias dos participantes. 
Descrições de dinâmicas interacionais envolvendo sujeitos com TEA, com maior ou menor comprometimento das funções interacionais e da linguagem, podem ser de grande importância para entendermos e descrevermos formas variadas de participação na interação. Análises nessa direção oferecem pistas de como sistematizar indicadores de competências ou limitações interacionais, sobretudo para aquelas interações com autistas não-verbais ou pouco verbais. Do ponto de vista interacional, alguns autores têm apontado para o fato de que fatores sociointeracionais situados, tais como, a familiaridade com o interlocutor, as posições corporais durante a interação, tópicos discursivos de maior ou menor interesse da criança, por exemplo, influenciam substancialmente na organização dessas interações (OCHS, 2010). Outra evidência da influência do trabalho interacional pode ser encontrada na literatura fonoaudiológica, que tem mostrado respostas positivas às intervenções fonoterapêuticas precoces propostas para as crianças (MARQUES, 1998; TAMANAHA; PERISSINOTO, 2011). Indicadores mais específicos, como contato visual, direcionamentos de olhar e gestos de apontar também têm sido descritos como relevantes, tanto em estudos clínicos (TAMANAHA; PERISSINOTO, 2019) quanto linguístico-interacionais (KORKIAKANGAS, 2014; DOAK, 2018) para entender qualitativamente e, portanto, com variabilidades as dinâmicas interacionais.

Uma consideração deve ser feita sobre a legibilidade da transcrição adotada. Reconhecemos que, ao mesmo tempo em que a granularidade da transcrição permite um aprofundamento analítico das temporalidades complexas das ações corporificadas e verbais nas interações, seu resultado final pode ser um texto com símbolos gráficos e formato pouco legíveis. A legibilidade da transcrição tem sido debatida por autores como Mondada (2003), Knoblauch et al. (2006), dentre outros. Da mesma forma, debate-se a possibilidade de tradução dos resultados da transcrição para além dos pesquisadores do campo dos estudos multimodais interacionais. Para o tratamento de corpora de interações envolvendo sujeitos com TEA, Doak (2018), por exemplo, tem proposto adaptar formas de representação da transcrição para tornar mais acessíveis os resultados analíticos da transcrição para leitores como professores, terapeutas e estudiosos de outras áreas, uma vez que as análises detalhadas das dinâmicas interacionais, viabilizadas pelo método analítico da transcrição multimodal, podem oferecer subsídios teóricos e mesmo práticos para profissionais que atuam com ou pesquisam TEA.

Por fim, consideramos que um empreendimento analítico que pudesse conjugar uma análise detalhada, como a apresentada aqui, a um universo maior de dados poderia ser pertinente, embora este não tenha sido o recorte realizado neste artigo. Reiteramos ainda que este tipo de análise nos pareceu produtiva para tratar interações das quais participam crianças autistas não-verbais, sinalizando futuros desdobramentos desta pesquisa. 


\section{Agradecimentos}

Esta pesquisa contou com financiamento da Fundação de Amparo à Pesquisa do Estado de São Paulo (FAPESP, Processo 2020/01199-9, Projeto "Ao mínimo gesto: estudo dos recursos multimodais (aspectos verbais, gestos, corpo e mundo material) nas interações envolvendo crianças com TEA (Transtornos do Espectro Autista)" e do CNPq (Processo 421937/2018-1 - Reconhecimento de padrão pré-verbal e verbal para predizer a necessidade de implementação do PECS em crianças com TEA).

\section{REFERÊNCIAS}

AUER, P. Projection in Interactionand Projection in Grammar. Text \& Talk: An Interdisciplinary Journal of Language, Discourse \& Communication Studies, v. 25, n. 1, p. 7-36, jan. 2005. Disponível em: https://doi.org/10.1515/text.2005.25.1.7. Acesso em: 15 set. 2020.

BAVELAS, J.; COATES, L.; JOHNSON, T. Listener Responses as a Collaborative Process: The Role of Gaze. Journal of Communication, v. 52, n. 3, p. 566-580, set. 2002. Disponível em: https://doi.org/10.1111/j.1460-2466.2002.tb02562.x. Acesso em: 20 set. 2020.

BAVELAS, J.; COATES, L.; JOHNSON, T. Listener Responses as a Collaborative Process: The Role of Gaze. In: ROSSANO, F. Gaze in Conversation. Oxford: Willey-Blackwell, 2013. p. 825.

CUNHA, G. R.; BORDINI, D.; CAETANO, S. C. Autismo, transtorno do espectro do autismo. In: CAETANO, S. C.; LIMA-HERNANDES, M. C.; PAULA, F. V.; RESENDE, B. D.; MÓDOLO, M. (org.). Autismo, Linguagem e Cognição. Jundiaí: Paco Editorial, 2015. p. 13-24.

DEPPERMANN, A. Turn-design at turn-beginnings: multimodal resources to deal with tasks of turn-construction in German. Journal of Pragmatics, v. 46, p. 91-121, jan. 2013. Disponível em: https://doi.org/10.1016/j.pragma.2012.07.010. Acesso em: 25 set. 2020.

DOAK, L. 'But I'd rather have raisins!': Exploring a hybridized approach to multimodal interaction in the case of a minimally verbal child with autism. Qualitative Research, v. 19, n. 1, p. 30-54, jan. 2018. Disponível em: https://doi:10.1177/1468794117752115. Acesso em: 12 set. 2020.

ERICKSON, F.; SCHULTZ, J. The counselor as gatekeeper. Social interaction in interviews. In: HAMMEL, E. Language, Thought and Culture: Advances in the Study of Cognition. New York: Academic Press, 1982. p. 237-260. 
GOODWIN, M.; CEKAITE, A. Orchestrating directive trajectories in communicative projects in family interaction. In: DREW, P.; COUPER-KUHLEN, E. (org.). Requesting in social interaction. Amsterdam: Benjamins, 2014. p. 181-210.

GOODWIN, M. H. Participation, affect, and trajectory in family directive/responsesequences. Text \& Talk: An Interdisciplinary Journal of Language, Discourse \& Communication Studies, v. 26, p. 513-541, set. 2006. Disponível em: https://doi.org/10.1515/TEXT.2006.021. Acesso em: 23 set. 2020.

GOODWIN, C. Gestures as a resource for the organization of mutual orientation. Semiotica, Amsterdam, v. 62, p. 29-49, jan. 1986. Disponível em: https://doi.org/10.1515/ semi.1986.62.1-2.51. Acesso em: 28 ago. 2020.

HEATH, C. Body Movement and Speech in Medical Interaction. Cambridge: Cambridge University Press, 1986.

KORKIAKANGAS, T.; RAE, J. The interactional use of eye-gaze in children with autism spectrum disorders. Interaction Studies, v. 15, n. 2, p. 233-259, 2014.

KEEVALLIK, L. The deictic nii 'so, in this way' in interaction. In: MONTICELLI, D.; PAJUSALU, R.; TREIKELDER, A. (org.). From utterance to uttering and vice versa. Multidisciplinary views on deixis. Tartu: University Press, 2005. p. 109-126.

KENDON, A. Gesture: Visible actions as utterance. Cambridge: Cambridge University Press, 2004.

KNOBLAUCH, H.; SCHNETTLER, B; JÜRGEN, R; HANS-GEORG, S. Video Analysis: Methodology and Methods. Qualitative Audiovisual Data Analysis in Sociology. 3. ed. Frankfurt: Peter Lang, 2006.

LAI, M. C.; LOMBARDO, M.; BARON-COHEN, S. Autism. The Lancet, v. 383, p. 896-910, set. 2013. Disponível em: https://doi.org/10.1016/S0140-6736(13)61539-1. Acesso em: 15 ago. 2020.

LEVINSON, S. C. Pragmática. São Paulo: WMF Martins Fontes, 2007.

LINDWALL, O.; EKSTRÖM, A. Instruction-in-Interaction: The Teaching and Learning of a Manual Skill. Human Studies, v. 35, p. 27-49, fev. 2012. Disponível em: https://doi. org/10.1007/s10746-012-9213-5. Acesso em: 23 set. 2020. 
LODER, L. L.; SALIMEN, P. G.; MULLER, M. Noções fundamentais: sequencialidade, adjacência e preferência. In: LODER, L. L.; JUNG, N. M. (org.). Fala-em-interação social: Introdução à Análise da Conversa Etnometodológica. Campinas: Mercado de Letras, 2008. p. 39-58.

MARCUSCHI, L. A. Análise da conversação. 2. ed. São Paulo: Ática, 1991.

MARQUES, C. Autismo - Intervenção terapêutica na $1^{\text {a }}$ infância. Análise Psicológica, Lisboa, v. 16, p. 139-144, mar. 1998. Disponível em: http://www.scielo.mec.pt/scielo. php?script=sci_arttext\&pid=S0870-82311998000100013. Acesso em: 20 ago. 2020.

MARLAIRE, C. L.; MAYNARD, D. W. Standardized testing as an interactional phenomenon. Sociology of Education, v. 63, n. 2, p. 83-101, abr. 1990. Disponível em: https://www.jstor. org/stable/2112856?seq=1. Acesso em: 26 set. 2020.

MONDADA, L. Transcribing silent actions: a multimodal approach of sequence organization. Social Interaction. Video-Based Studies of Human Sociality, v. 2, n. 1, mar. 2019. Disponível em: https://doi.org/10.7146/si.v2i1.113150. Acesso em: 20 set. 2020.

MONDADA, L. Challenges of multimodality: language and the body in social interaction. Journal of Sociolinguistics, v. 20, n. 3, p. 336-366, jun. 2016a. Disponível em: https://doi. org/10.1111/josl.1_12177. Acesso em: 20 set. 2020.

MONDADA, L. Página Pessoal. Conventions for multimodal transcription, versão3.0.6. jul. 2016b. Disponível em: https://franzoesistik.philhist.unibas.ch/fileadmin/user_upload/ franzoesistik/mondada_multimodal_conventions.pdf. Acesso em: 10 ago. 2020.

MONDADA, L. Instructions in the operating room: How surgeons direct their assistant's hands. Discourse Studies, v. 16, n. 2, p. 131-161, mar. 2014a. Disponível em: https://doi. org/10.1177/1461445613515325. Acesso em: 20 set. 2020.

MONDADA, L. The local constitution of multimodal resources for social interaction. Journal of Pragmatics, v. 65, p. 137-156, maio 2014b. Disponível em: https://doi.org/10.1016/j. pragma.2014.04.004. Acesso em: 20 set. 2020.

MONDADA, L. Understanding as an embodied, situated and sequential achievement in interaction. Journal of Pragmatics, v. 43, n. 2, p. 542-552, jan. 2011. Disponível em: https:// doi.org/10.1016/j.pragma.2010.08.019. Acesso em: 20 set. 2020. 
MONDADA, L. The embodied and negotiated production of assessments in instructed actions. Research on Language and Social Interaction, v. 42, n. 4, p. 329-361, nov. 2009. Disponível em: https://doi.org/10.1080/08351810903296473. Acesso em: 18 set. 2020.

MONDADA, L. Multimodal resources for turn-taking. Discourse Studies, v. 9., p. 194-225, 2007. Disponível em: https://doi.org/10.1177/1461445607075346. Acesso em: 07 fev. 2021.

MONDADA, L. Temporalité, séquentialité et multimodalitéau fondement del'organisation de I'interaction: Le pointage comme pratique de prise du tour. Cahiers de Linguistique Française, v. 26, p. 269-292, ago. 2004. Disponível em: https://clf.unige.ch/files/4514/4102/7607/13Mondada_nclf26.pdf. Acesso em: 22 ago. 2020.

MONDADA, L. Working with video: how surgeons produce video records of their actions. Visual Studies, v. 18, n. 1, p. 58-73, jun. 2003. Disponível em: https://doi.org/10.1080/1472 586032000100083. Acesso em: 20 set. 2020.

OCHS, E.; SOLOMON, O. Autistic Sociality. Ethos, v. 38, n. 1, p. 69-92, mar. 2010. Disponível em: https://doi.org/10.1111/j.1548-1352.2009.01082.x. Acesso em: 25 ago. 2020.

OLOFF, F. Embodied withdrawal after overlap resolution. Journal of Pragmatics, v. 46, n. 1, p. 139-156, jan. 2013. Disponível em: https://doi.org/10.1016/j.pragma.2012.07.005. Acesso em: 22 set. 2020.

ROSSANO, F. Gaze in conversation. In: SIDNELL, J.; STIVERS, T. (org.). The Handbook of Conversation Analysis. Nova Jersey: Wiley-Blackwell, 2012.

TAMANAHA, A. C.; PERISSINOTO, J. Transtornos do Espectro do Autismo Implementando Estratégias para a Comunicação. São Paulo: Book Toy, 2019.

SACKS, H.; SCHEGLOFF, E. A.; JEFFERSON, G. A simplest systematic for the organization of turn-taking for conversation. Language, Baltimore, v. 50, n. 4, p. 696-735, 1974. Tradução A. M. S. da Cunha et al. Veredas, Juiz de Fora, v 7, n. 1, p. 9-73, dez. 2003. Disponível em: https://www.ufjf.br/revistaveredas/files/2009/12/artigo14.pdf. Acesso em: 20 set. 2020.

SILVA, C. R.; ANDRADE, D. N. P.; OSTERMANN, A. C. Análise da Conversa: uma breve introdução. ReVEL, v. 7, n. 13, p. 1-21, ago. 2009. Disponível em: http://www.revel.inf.br/ files/artigos/revel_13_analise_da_conversa.pdf. Acesso em: 23 set. 2020. 
STREECK, J.; HARTGE, U. Previews: gestures at the transition place. In: AUER, P.; DI LUZIO, A. (org.). The contextualization of language. Amsterdam: Benjamins, 1992. p. 135-158.

STREECK, J.; KNAPP, M. L. The interaction of visual and verbal features in human communication. In: POYATOS, F. Advances in Non-Verbal Communication. Amsterdam: John Benjamins, 1992. p. 3-23.

STUKENBROCK, A. Take the words out of my mouth: Verbal instructions as embodied practices. Journal of Pragmatics, v. 65, p. 80-102, maio 2014, Disponível em: https://doi. org/10.1016/j.pragma.2013.08.017. Acesso em: 23 set. 2020.

TAMANAHA, A. C.; PERISSINOTO, J. Comparação do processo evolutivo de crianças do espectro autístico em diferentes intervenções terapêuticas fonoaudiológicas. Jornal da Sociedade Brasileira de Fonoaudiologia, v. 23, n. 1, p. 8-12, jan. 2011. Disponível em: https://doi.org/10.1590/S2179-64912011000100005. Acesso em: 23 set. 2020.

WITTENBURG, P.; BRUGMAN, H.; RUSSEL, A.; KLASSMANN, A.; SLOETJES, H. ELAN: A professional framework for multimodality research. Proceedings of the Fifth International Conference on Language Resources and Evaluation. Genoa: European Language Resources Association, 2006. Disponível em: http://www.Irec-conf.org/proceedings/lrec2006/ pdf/153_pdf.pdf. Acesso em: 20 set. 2020. 